\title{
Multi-Location Evaluation of Yield and Yield Related Trait Performance in Food Barley (Hordeum Vulgare L.) Genotypes at Western Oromia, Ethiopia
}

\author{
Geleta Negash $^{1 *}$, Wagar Raga ${ }^{2}$, Biru Alemu \\ ${ }^{1,2}$ Oromia Agricultural Research Institute (IQQO), Haro Sabu Agricultural Research Center (HSARC), P.O.Box \\ 10, Kellem Wollega, Dembi Dollo
}

*Corresponding Author: Geleta Negash, Oromia Agricultural Research Institute (IQQO), Haro Sabu Agricultural Research Center (HSARC), P.0.Box 10, Kellem Wollega, Dembi Dollo

\begin{abstract}
Barley is one of the founders, old world agriculture and the first domesticated cereal crop. This experiment was conducted on fifteen barley genotypes against checks at Haro Sabu Agricultural Research Center sub site in 2017-2018.The objective was to assess the magnitude of genotype by environment interaction, grain yield stability, high yielding, insect pest tolerant genotypes in the growing areas of western Oromia. Seeds were planted in RCBD with three replications. Eleven agronomic traits and four disease reaction data were evaluated. Analysis of variance detected significant difference among genotypes for most observed traits both separated and combined analysis. Observation attained significant differences over years and locations for almost all traits. The combine ANOVA and the AMMI analysis for grain yield across environments revealed significantly affected by environments, that hold $41 \%$ of the total variation.The genotype and genotype by environmental interation, were significant and accounted for $10.46 \%$ and $27.69 \%$ respectively. Pricipal component 1 and 2 accounted for $15.74 \%$ and $5.71 \%$ of the GEI respectively with a total of $21.45 \%$ variation.In general, G2 and G12 were identified as the best genotypes for yielding ability, stability, tolerant to diseases and use as parents in future breeding programs.
\end{abstract}

Keywords: AMMI, food barley, GGEI, performance, stability

Abbreviations: $A M M I=$ additive main effects and multiplicative interactions, ANOVA= analysis of variance, $G E I$ = genotype by enviromental interaction, GGEI= genotype and genotype by enviromental interaction, $R C B D=$ Randomized Completed Block Design

\section{INTRODUCTION}

Barley (Hordeum vulgare L) $(2 \mathrm{n}=2 \mathrm{x}=14)$ is one of the most important staple food crops in the highlands of Ethiopia. It is a cool season crop, the most dependable and early maturing cereal grain with relatively high yield potential including in marginal areas where other cereal crops cannot have adapted [14, 31]. The major barley production areas of the world include: Europe, the Mediterranean fringe of North Africa, Ethiopia and the Middle East, former USSR, China, India, Canada and USA [16]. Ethiopia is the second largest barley producer in Africa, next to Morocco, accounting for about $25 \%$ of the total barley production in the continent [10]. However, there is great yield gap between national average yield $\left(2.11 \mathrm{t} / \mathrm{ha}^{-1}\right)$ [7] and world average yield $\left(3.04 \mathrm{t} / \mathrm{ha}^{-1}\right)$ (Foreign Agricultural Service/USDA April 2017 Office of Global Analysis). This production limitation may be attributed to primarily the low yielding ability of farmers' cultivars, which are the dominant varieties in use; the influence of several biotic and a biotic stresses; and poor promotion of improved barley production package technologies [1].

Environmental fulactuation and interaction is also the major limitation for food barley production and productivity. Genotype by environment interaction (GEI) is the differential responses of different genotypes across a range of environments [29]. In breeding, genotype $\mathrm{x}$ environmental interaction ( $\mathrm{G} \mathrm{x}$ E) cause many difficulties, while the environmental factors such as temperature, soil affect the performance of genotypes. Genotype $x$ environment (GE) interaction reduces the genetic progress in plant breeding programs through minimizing the association between phenotypic and genotypic values [6].Consequently, multi-environment yield trials are essential in assessing of genotype by environment 
interaction (GEI) and identification of superior genotypes in the final selection cycles [30, 32]. Phenotypes are a mixture of genotype $(\mathrm{G})$ and environment $(\mathrm{E})$ components and interactions $(\mathrm{G} \times \mathrm{E})$ between them.GxE interactions complicate process of selecting genotypes with superior performance. Therefore, multi-environment trails (METs) are widely used by plant breeders to evaluate the relative performance of genotypes for target environments [9].

The additive main effects and multiplicative interaction (AMMI) model have also led to more understanding in the complicated patterns of genotypic responses to the environment [13]. These patterns have been successfully related to biotic and abiotic factors. [20], proposed another methodology known as GGE-biplot for graphical display of GE interaction pattern of MET data with many advantages. GGE biplot is an effective method based on principal component analysis (PCA) which fully explores MET data. It allows visual examination of the relationships among the test environments, genotypes and the GE interactions. The first two principle components (PC1 and PC2) are used to produce a two dimensional graphical display of genotype by environment interaction (GGE-biplot). If a large portion of the variation is explained by these components, a rank-two matrix, represented by a GGE- biplot, is appropriate [21].

The objective of this study was to assess genotype performances, the magnitude of genotype $\mathrm{x}$ environment interaction for grain yield and to evaluate the stability for grain yield and tolerant to diseases of food barley genotypes.

\section{MATERIALS AND MeTHODS}

\subsection{Description of Locations}

The experiment was conducted at three different rain fed locations in Kellem and west Wollega zones of Haro-sebu agricultural research center at Belem research sub site (altitude 1759 masl, 09 $02^{\circ} \mathrm{N}$,

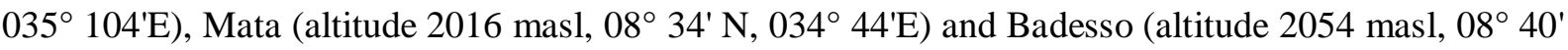
$\mathrm{N}, 034^{\circ} 47^{\prime} \mathrm{E}$ ) in western Oromia, Ethiopia, during the 2017-2018 main cropping season, that represent the varying agro ecologies of the barley growing areas of the zones.

\subsection{Experimental Materials}

Fifteen regional variety trial of food barley genotypes, together with three checks, two standard checks (Abdane and Dinsho) and one farmer's varieties, were included in the trial (Table 1). The genotypes were selected based on average performance and agro-ecological adaptation. Genotypes were obtained from Ethiopia biodiversity institute (EBI) Sinana Agricultural Research Center and from farmers for the farmers' varieties.

Table1. List of food barley genotypes evaluated in 2017-2018 main cropping season

\begin{tabular}{|c|c|c|c|}
\hline No & Codes & Genotypes Acc.No & Sources \\
\hline 1 & G1 & 242573 & EBI \\
\hline 2 & G2 & 220677 & EBI \\
\hline 3 & G3 & Abdane & SARC \\
\hline 4 & G4 & 202820 & EBI \\
\hline 5 & G5 & 237021 & EBI \\
\hline 6 & G6 & 219142 & EBI \\
\hline 7 & G7 & 241675 & Farmer \\
\hline 8 & G8 & Local & EBI \\
\hline 9 & G9 & 225176 & EBI \\
\hline 10 & G10 & 4560 & EBI \\
\hline 11 & G11 & 233028 & EBI \\
\hline 12 & G12 & 219307 & EBI \\
\hline 13 & G13 & 64260 & SARC \\
\hline 14 & G14 & 233030 & Dinsho \\
\hline 15 & G15 & & S \\
\hline
\end{tabular}

G-genotype, Acc. No- accession number EBI- Ethiopia biodiversity institute, SARC- Sinana Agricultural Reaserch center 


\subsection{Experimental Design and Management}

Randomized completed block design (RCBD) with three replications was used in all locations. Each experimental plot had six rows of $2.5 \mathrm{~m}$ long spaced and $20 \mathrm{~cm}$ apart with a plot area of $1.2 \mathrm{~m} \times 2.5 \mathrm{~m}$. Drill planting by hand was used with the same rate for all locations. Fertilizer was applied at a rate of 41 and $46 \mathrm{~kg} \mathrm{ha}^{-1}$ of $\mathrm{N}$ and $\mathrm{P}_{2} \mathrm{O}_{5}$, respectively, in the form of Urea and DAP. All $\mathrm{P}_{2} \mathrm{O}_{5}$ and half of $\mathrm{N}$ were applied during planting, while the rest half splits were applied at tillering stages. A seeding rate of $85 \mathrm{~kg} \mathrm{ha}^{-1}$ was used. First weeding was carried out 35 days after emergence and the second one at 30 days after the first weeding. Weeding was done up to four times for all locations. The data considered for analysis was from the candidates of the net plot, thus the four central harvestable rows. The harvested genotypes were sundried before being tested for moisture content where $12 \%$ was the preferred average moisture content using moisture tester. Grain yield data was then obtained by weighing the dried grain using a digital scale.

\subsection{Data Collection Method}

Ten plants were selected randomly before heading from each row (four harvestable rows) and tagged with thread and all the necessary plant based data were collected from these sampled plants.

\subsection{Plot Basis}

The following plant parameters were determined: Days to heading (DH), Days to maturity (DM), Lodging percentage (LDG), Thousand seed weight (TSW), Grain yield (GY), and the four major economically important food barley diseases such as scald, septoria (SEP), stem rust (SR), and leaf rust (LR)

\subsection{Plant Basis}

Plant height (PH), Productive tillers (PTR), Spike length (SL), Grain per spike (GPS), spike weight per plant (SWPP) and number of spike lets per spike (NSPS)

\section{Statistical ANalysis}

\subsection{Analysis of Variance is Calculated using the Model}

$\mathrm{Yij}=\mu+\mathrm{Gi}+\mathrm{Ej}+\mathrm{GEij}$

Where Yij is the corresponding variable of the $\mathrm{i}^{- \text {th }}$ genotype in $\mathrm{j}^{- \text {th }}$ environment, $\mu$ is the total mean, Gi is the main effect of $i^{\text {th }}$ genotype, Ej is the main effect of $j^{\text {th }}$ environment, GEij is the effect of genotype $\mathrm{x}$ environment interaction.

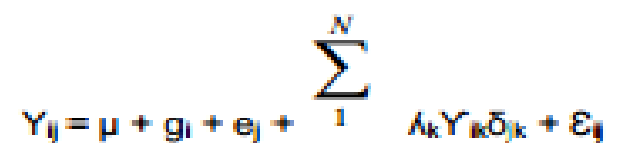

\subsection{The AMMI Model used was}

Where Yij is the grain yield of the $\mathrm{i}^{\text {th }}$ genotype in the $\mathrm{j}^{\text {th }}$ environment, $\mu$ is the grand mean, gi and ej are the genotype and environment deviation from the grand mean, respectively, $K \mathrm{k}$ is the eigenvalue of the principal component analysis (PCA) axis $\mathrm{k}$, Yik and $\delta \mathrm{jk}$ are the genotype and environment principal component scores for axis $\mathrm{k}, \mathrm{N}$ is the number of principal components retained in the model, and Eij is the residual term.

GGE-biplot methodology, which is composed of two concepts, the biplot concept [12] and the GGE concept [20], was used to visually analyze the METs data. This methodology uses a biplot to show the factors ( $\mathrm{G}$ and $\mathrm{GE}$ ) that are important in genotype evaluation and that are also the source of variation in GEI analysis of METs data [22]. The GGE-biplot shows the first two principal components derived from subjecting environment centered yield data (yield variation due to GGE) to singular value decomposition [20].AMMI Stability Value (ASV): ASV is the distance from the coordinate point to the origin in a two-dimensional plot of IPCA1 scores against IPCA2 scores in the AMMI model [24]. Because the IPCA1 score contributes more to the GxE interaction sum of squares, a weighted value is needed. This weighted value was calculated for each genotype and each environment according to the relative contribution of IPCA1 to IPCA2 to the interaction sum of squares as follows: 


$$
A S V=\sqrt{\left[\left(S S_{I P C A 1} \div S S_{I P C A 2}\right)(\text { IPCA1score })\right]^{2}+(\text { IPCA2score })^{2}}
$$

Where, $\mathrm{SS}_{\mathrm{IPCA} 1} / \mathrm{SS}_{\mathrm{IPCA} 2}$ is the weight given to the IPCA1-value by dividing the IPCA1 sum of squares by the IPCA2 sum of squares. The larger the ASV value, either negative or positive, the more specifically adapted a genotype is to certain environments. Smaller ASV values indicate more stable genotypes across environments [24]. Genotype Selection Index (GSI): Stability is not the only parameter for selection as most stable genotypes would not necessarily give the best yield performance. Therefore, based on the rank of mean grain yield of genotypes (RYi) across environments and rank of AMMI stability value RASVi), genotype selection index (GSI) was calculated for each genotype as:

$$
\text { GSIi }=\text { RASVi }+ \text { RYi }
$$

A genotype with the least GSI is considered as the most stable [11]. Analysis of variance was carried out using statistical analysis system (SAS) version 9.2 software [25]. Additive Main Effect and Multiplicative Interaction (AMMI) analysis and GGE bi-plot analysis were performed using Gen Stat 15th edition statistical package [19]

\section{RESUlTS AND DISCUSSIONS}

\subsection{Combined Analysis of Variance}

The mean square of analysis of variance (ANOVA) is presented in Table 2. Highly significant differences were detected among the main and the interaction effects $(\mathrm{P} \leq 0.01)$ for most of the parameters. The combined analysis of variance revealed that significant differences were recorded across location for all parameters except spike weight per plant. Year*genotypes effects were significant for most traits. Year*location *genotypes were significant for most traits such as days to heading, days to maturity, stem and leaf rust, spike length, grain per spike, thousand seed weight and grain yield.

Table2. Analysis of variance (ANOVA) for grain yield and yield related traits of food barley genotypes evaluated in 2017-2018 main cropping season

\begin{tabular}{|c|c|c|c|c|c|c|c|c|c|}
\hline Source & DF & DH & DM & PTL & Scald & SEP & SR & LR & LDG \\
\hline rep & 2 & 20.69 & 12.51 & 1.51 & 0.31 & 0.08 & 0 & 0.06 & 0.01 \\
\hline Geno. & 14 & $163.17^{* *}$ & $163.73^{* *}$ & $2.20^{* *}$ & 0.3 & $0.17^{* *}$ & $0.34^{* *}$ & $0.36^{* *}$ & $0.66^{*}$ \\
\hline loc & 2 & $371.83^{* *}$ & $179.45^{* *}$ & $24.35^{* *}$ & $9.08^{* *}$ & $8.81^{* *}$ & $17.68^{* *}$ & $20.38^{* *}$ & $16.77^{* *}$ \\
\hline yr & 1 & $2881.20^{* *}$ & 2.7 & $138.96^{* *}$ & $3.91^{* *}$ & $1.07^{* *}$ & $2.22^{* *}$ & $2.80^{* *}$ & $47.71^{* *}$ \\
\hline geno*loc & 28 & $22.81^{*}$ & $33.77^{* *}$ & 0.6 & $0.78^{* *}$ & $0.08^{*}$ & $0.21^{* *}$ & $0.21^{* *}$ & 0.44 \\
\hline geno*yr & 14 & $120.10^{* *}$ & $104.67^{* *}$ & $1.66^{*}$ & 0.25 & 0.04 & $0.09^{* *}$ & 0.1 & $0.56^{*}$ \\
\hline loc*yr $^{*}$ & 2 & $124.34^{* *}$ & 15.63 & $16.23^{* *}$ & $3.91^{* *}$ & $1.07^{* *}$ & $2.22^{* *}$ & $2.80^{* *}$ & $1.17^{*}$ \\
\hline geno*loc*yr $^{*}$ & 28 & $28.30^{*}$ & $34.11^{* *}$ & 0.61 & 0.25 & 0.04 & $0.09^{* *}$ & $0.10^{*}$ & 0.38 \\
\hline
\end{tabular}

Table2. Continue

\begin{tabular}{|c|c|c|c|c|c|c|c|c|}
\hline Source & DF & PH & SL & SWPP & SPS & GPS & TSW & QTHA \\
\hline rep & 2 & 28.83 & 0.2 & 0.35 & 54.74 & 3.53 & 21.29 & 13.66 \\
\hline geno. & 14 & $209.42^{* *}$ & $2.06^{*}$ & 0.24 & $61.22^{* *}$ & $126.71^{* *}$ & $246.85^{*}$ & $823.16^{* *}$ \\
\hline loc & 2 & $4308.57^{* *}$ & $2.60^{*}$ & 0.69 & $217.74^{* *}$ & $119.41^{*}$ & $2933.84^{* *}$ & $851.03^{* *}$ \\
\hline $\mathbf{y r}$ & 1 & $931.86^{* *}$ & 0.02 & 0.4 & $84.90^{*}$ & $311.80^{* *}$ & $8079.74 * *$ & $9684.87^{* *}$ \\
\hline geno*loc & 28 & 51.28 & 0.99 & 0.42 & 18.95 & $48.69^{*}$ & 155.8 & $62.89 * *$ \\
\hline geno*yr $^{*}$ & 14 & $133.98^{* *}$ & $1.58^{*}$ & 0.39 & $36.70^{*}$ & $101.68^{* *}$ & 139.09 & $81.29^{*}$ \\
\hline loc*yr $^{*} \mathbf{y r}$ & 2 & $1603.92^{* *}$ & $3.55^{*}$ & 0.94 & $88.44^{*}$ & $1264.39^{* *}$ & $3855.68^{* *}$ & $1132.38^{* *}$ \\
\hline geno*loc*yr $^{*}$ & 28 & 79.04 & $1.46^{*}$ & 0.46 & 24.58 & $59.00^{* *}$ & $176.35^{*}$ & $72.41^{*}$ \\
\hline
\end{tabular}

Key: ***, significant at $5 \%$ and $1 \%$ respectively, Loc *geno $=$ location by genotype, $Y{ }^{*}$ Loc ${ }^{*}$ geno $=$ year by location by genotype, DF -degree of freedom, DH-Days to Heading; DM-Days to Maturity; PTL- productive tillers, SEP-septoria, SR-stem rust, LR- leaf rust, LDG-lodging, PH-Plant Height; SL- spike Length; SWPPSpike Weight per plant, SPS-spike lets per spike, GPS-Grain per spike, TSW-Thousand Seed Weight, QTHAYield quintal per Hectare.

\subsection{Yield Performance of Food Barley Genotypes Across Locations}

Mean performance for grain yield across location and year presented in Table 3. Study by [18] indicated that genotypes constantly performed best in some environments and fluctuating across some 
Multi-Location Evaluation of Yield And Yield Related Trait Performance In Food Barley (Hordeum Vulgare L.) Genotypes at Western Oromia, Ethiopia

locations. Accordingly, the average grain yield ranged from the lowest of $19.33 \mathrm{Qtha}^{-1}$ at Bedesso sub site in 2017 to the highest of 36.47 Qtha $^{-1}$ at Belem site in 2018 with grand mean of 29.29 Qtha $^{-1}$.

The average grain yields across environments ranged from the lowest of $23.5 \mathrm{Qtha}^{-1}$ for G9 to the highest of 37.5 Qtha $^{-1}$ for G2. This wide variation might be due to the genetic potential of the different genotypes. Therefore, G2 and G12 were constantly performed across the locations. The difference in yield rank of genotypes across the environments exhibited the high crossover type of genotypes $\mathrm{x}$ environmental interaction $[22,3]$.

Table3. Mean grain yield $\left(Q_{\text {tha }}{ }^{-1}\right)$ of food barley genotypes evaluated at three environments

\begin{tabular}{|c|c|c|c|c|c|c|c|}
\hline \multicolumn{8}{|c|}{ Grain Yield in Qtha $^{-1}$} \\
\hline \multirow[b]{2}{*}{ Genotype } & \multicolumn{3}{|c|}{2017} & \multicolumn{3}{|c|}{2018} & \multirow[b]{2}{*}{ Com.mean } \\
\hline & Belem & Bedesso & Mata & Mata & Belem & Bedesso & \\
\hline Dinsho & $27.88 \mathrm{bc}$ & $14.71 \mathrm{fg}$ & $22.043 \mathrm{fg}$ & $35.27 \mathrm{~b}$ & $31.92 \mathrm{def}$ & $32.98 \mathrm{~b}$ & $27.5 \mathrm{def}$ \\
\hline G1 & $31.23 \mathrm{ab}$ & $16.56 \mathrm{~d}-\mathrm{g}$ & $29.35 b-e$ & $31.69 \mathrm{~b}$ & $35.55 \mathrm{cde}$ & $30.18 \mathrm{~b}$ & $29.1 \mathrm{cde}$ \\
\hline G10 & $25.19 \mathrm{~b}-\mathrm{e}$ & $20.17 b-f$ & $21.55 \mathrm{fg}$ & $35.06 \mathrm{~b}$ & $33.59 c-f$ & 30.197 & $27.6 \mathrm{def}$ \\
\hline G11 & $21.45 \mathrm{def}$ & $23.03 \mathrm{bcd}$ & $29.61 b-e$ & $28.92 \mathrm{~b}$ & $29.143 \mathrm{ef}$ & $29.80 \mathrm{~b}$ & 27.0efg \\
\hline G12 & $17.87 \mathrm{fgh}$ & $26.85 a b$ & $30.81 \mathrm{a}-\mathrm{d}$ & $37.73 \mathrm{ab}$ & $43.94 \mathrm{abc}$ & $46.95 a$ & $34.0 \mathrm{ab}$ \\
\hline G13 & $14.58 \mathrm{gh}$ & $22.02 b-f$ & 23.38 efg & $29.74 b$ & $30.85 \mathrm{def}$ & $31.43 \mathrm{~b}$ & $25.3 \mathrm{gh}$ \\
\hline Abdane & $7.79 \mathrm{i}$ & $12.35 \mathrm{~g}$ & $36.94 \mathrm{a}$ & $42.66 \mathrm{ab}$ & $40.88 \mathrm{a}-\mathrm{d}$ & $37.21 \mathrm{~b}$ & $29.6 \mathrm{cde}$ \\
\hline G14 & $34.60 \mathrm{a}$ & $31.38 \mathrm{a}$ & $29.41 \mathrm{~b}-\mathrm{e}$ & $33.34 \mathrm{~b}$ & $24.87 \mathrm{f}$ & $30.72 b$ & $30.7 \mathrm{bcd}$ \\
\hline G2 & $23.07 \mathrm{c}-\mathrm{f}$ & $24.52 \mathrm{abc}$ & 24.99def & $49.86 \mathrm{a}$ & $50.48 \mathrm{a}$ & $51.89 \mathrm{a}$ & $37.5 \mathrm{a}$ \\
\hline G4 & $30.72 \mathrm{ab}$ & 22.76b-e & $31.54 \mathrm{a}-\mathrm{d}$ & $31.13 \mathrm{~b}$ & $31.72 \mathrm{def}$ & $30.38 b$ & $29.7 \mathrm{cde}$ \\
\hline G5 & $26.59 \mathrm{bcd}$ & $15.10 \mathrm{fg}$ & $34.74 a b c$ & $31.95 \mathrm{~b}$ & $46.37 \mathrm{ab}$ & $34.98 \mathrm{~b}$ & $31.6 \mathrm{bc}$ \\
\hline G6 & $29.55 \mathrm{ab}$ & $16.11 \mathrm{~d}-\mathrm{g}$ & $35.31 \mathrm{ab}$ & $30.75 b$ & $35.81 \mathrm{cde}$ & $30.13 b$ & $29.6 \mathrm{cde}$ \\
\hline G7 & $19.66 \mathrm{efg}$ & $15.31 \mathrm{efg}$ & $33.48 \mathrm{abc}$ & $37.88 \mathrm{ab}$ & $34.60 \mathrm{c}-\mathrm{f}$ & $29.66 b$ & $28.4 c-f$ \\
\hline G9 & $12.96 \mathrm{hi}$ & $11.69 \mathrm{~g}$ & $16.58 \mathrm{~g}$ & $32.88 \mathrm{~b}$ & $39.10 \mathrm{~b}-\mathrm{e}$ & $27.50 \mathrm{~b}$ & $23.5 \mathrm{~g}$ \\
\hline Local & $14.36 \mathrm{gh}$ & $17.47 \mathrm{c}-\mathrm{g}$ & $28.203 c-f$ & $36.48 \mathrm{ab}$ & $38.19 \mathrm{~b}-\mathrm{e}$ & $34.34 \mathrm{~b}$ & $28.2 \mathrm{c}-\mathrm{f}$ \\
\hline Mean & 22.50 & 19.33 & 28.53 & 35.02 & 36.47 & 33.89 & 29.29 \\
\hline CV \% & 16.32 & 23.26 & 14.84 & 23.53 & 17.14 & 17.16 & 19.33 \\
\hline $\mathbf{R}^{2}$ & 0.88 & 0.71 & 0.74 & 0.41 & 0.64 & 0.68 & 79.3 \\
\hline LSD 5\% & 6.14 & 7.52 & 7.08 & 13.78 & 10.46 & 9.73 & 3.724 \\
\hline F-test & $* *$ & $* *$ & $* *$ & *** & $* *$ & *** & $* *$ \\
\hline
\end{tabular}

Key: G-genotype, R2, R-squire, CV-coefficient of variation, LSD-least significant different.

\subsection{Agronomic Performance}

Combined mean grain yield and other agronomic traits are presented in Table 4. Genotype (G12) was recorded medium days to heading, days to maturity, and plant height, indicated that, the possibility to develop resistant variety against lodging problems. And also it recorded the highest productive tillers per plant, thousand seed weight and grain yield. Similarly, Genotype (G2) was recorded medium days to heading, days to maturity, productive tillers and thousand seed weight and the highest spike length. These recommend great flexibility for developing improved varieties appropriate for different agroecologies with variable length of growing period and high in grain yield status. Moreover, these genotypes (G12 and G2) were recorded the highest grain yield and they had $14.95 \%$, and $26.58 \%$ yield advantage over the best standard check (Abdane) respectively.

Table4. Combined mean grain yield and other agronomic traits of food barley genotypes

\begin{tabular}{|c|c|c|c|c|c|c|}
\hline Gen/Vrt & DH & DM & PTL & LDG & PH & SL \\
\hline Dinsho & $57.4 \mathrm{~cd}$ & $89.6 \mathrm{de}$ & $3.5 \mathrm{~b}-\mathrm{e}$ & $2.0 \mathrm{ab}$ & $90.2 \mathrm{a}-\mathrm{e}$ & $8.4 \mathrm{bcd}$ \\
\hline G1 & $54.3 \mathrm{ef}$ & $89.0 \mathrm{def}$ & $3.5 \mathrm{~b}-\mathrm{e}$ & $1.9 \mathrm{abc}$ & $90.6 \mathrm{a}-\mathrm{d}$ & $8.5 \mathrm{abc}$ \\
\hline G10 & $55.8 \mathrm{de}$ & $90.4 \mathrm{cde}$ & $3.8 \mathrm{a}-\mathrm{d}$ & $2.1 \mathrm{a}$ & $92.8 \mathrm{a}-\mathrm{d}$ & $8.0 \mathrm{~cd}$ \\
\hline G11 & $56.3 \mathrm{cde}$ & $92.6 \mathrm{bc}$ & $3.9 \mathrm{abc}$ & $1.5 \mathrm{~cd}$ & $89.5 \mathrm{~b}-\mathrm{e}$ & $8.9 \mathrm{ab}$ \\
\hline G12 & $58.8 \mathrm{bc}$ & $94.3 \mathrm{ab}$ & $4.4 \mathrm{a}$ & $1.7 \mathrm{bcd}$ & $93.6 \mathrm{abc}$ & $8.7 \mathrm{ab}$ \\
\hline G13 & $60.5 \mathrm{~b}$ & $96.4 \mathrm{a}$ & $3.1 \mathrm{e}$ & $1.9 \mathrm{abc}$ & $89.5 \mathrm{~b}-\mathrm{e}$ & $8.4 \mathrm{bcd}$ \\
\hline Abdane & $64.2 \mathrm{a}$ & $96.5 \mathrm{a}$ & $3.6 \mathrm{~b}-\mathrm{e}$ & $1.5 \mathrm{~d}$ & $82.9 \mathrm{f}$ & $7.9 \mathrm{~d}$ \\
\hline G14 & $54.9 \mathrm{de}$ & $90.7 \mathrm{cde}$ & $3.9 \mathrm{abc}$ & $1.6 \mathrm{~cd}$ & $94.6 \mathrm{ab}$ & $8.7 \mathrm{ab}$ \\
\hline G2 & $57.2 \mathrm{~cd}$ & $93.2 \mathrm{~b}$ & $3.9 \mathrm{ab}$ & $1.8 \mathrm{a}-\mathrm{d}$ & $90.3 \mathrm{a}-\mathrm{d}$ & $9.1 \mathrm{a}$ \\
\hline G4 & $56.8 \mathrm{cde}$ & $90.8 \mathrm{~cd}$ & $4.0 \mathrm{ab}$ & $1.7 \mathrm{bcd}$ & $91.0 \mathrm{a}-\mathrm{d}$ & $8.6 \mathrm{ab}$ \\
\hline G5 & $55.4 \mathrm{de}$ & $88.4 \mathrm{ef}$ & $3.2 \mathrm{de}$ & $2.0 \mathrm{ab}$ & $87.7 \mathrm{def}$ & $9.1 \mathrm{a}$ \\
\hline G6 & $54.6 \mathrm{e}$ & $90.2 \mathrm{de}$ & $3.3 \mathrm{cde}$ & $1.6 \mathrm{~cd}$ & $88.4 \mathrm{cde}$ & $8.5 \mathrm{bcd}$ \\
\hline G7 & $56.8 \mathrm{cde}$ & $86.9 \mathrm{f}$ & $3.5 \mathrm{~b}-\mathrm{e}$ & $1.6 \mathrm{~cd}$ & $94.8 \mathrm{a}$ & $8.4 \mathrm{bcd}$ \\
\hline G9 & $60.5 \mathrm{~b}$ & $93.3 \mathrm{~b}$ & $3.8 \mathrm{abc}$ & $1.8 \mathrm{a}-\mathrm{d}$ & $85.0 \mathrm{ef}$ & $8.4 \mathrm{bcd}$ \\
\hline Local & $52.0 \mathrm{f}$ & $87.1 \mathrm{f}$ & $4.0 \mathrm{ab}$ & $1.9 \mathrm{abc}$ & $93.7 \mathrm{ab}$ & $8.5 \mathrm{abc}$ \\
\hline M \pm SEM & $57.03 \pm 0.41$ & $91.29 \pm 0.34$ & $3.69 \pm 0.08$ & $1.78 \pm 0.05$ & $90.31 \pm 0.67$ & $8.54 \pm 0.06$ \\
\hline CV\% & 6.76 & 3.74 & 25.01 & 31.54 & 8.78 & 10.75 \\
\hline
\end{tabular}


Multi-Location Evaluation of Yield And Yield Related Trait Performance In Food Barley (Hordeum Vulgare L.) Genotypes at Western Oromia, Ethiopia

\begin{tabular}{|c|c|c|c|c|c|c|}
\hline \multicolumn{7}{|c|}{} \\
\hline $\mathrm{R}^{2} \%$ & 78 & 75 & 67 & 69 & 66 & 47 \\
\hline LSD 5\% & 2.54 & 2.25 & 0.61 & 0.37 & 5.22 & 0.6 \\
\hline F test & $* *$ & $* *$ & $* *$ & $* *$ & $* *$ & $* *$ \\
\hline
\end{tabular}

Table4. continue

\begin{tabular}{|c|c|c|c|c|c|c|}
\hline Gen/Vrt & SWPP & SPS & GPS & TSW & QTHA & YLD AV \\
\hline Dinsho & $1.3 \mathrm{~b}$ & $17.38 \mathrm{bcd}$ & $24.06 \mathrm{cde}$ & $46.61 \mathrm{ab}$ & $27.5^{\text {def }}$ & -7.09 \\
\hline G1 & $1.42 \mathrm{ab}$ & $18.41 \mathrm{a}-\mathrm{d}$ & $25.96 \mathrm{bcd}$ & $39.97 \mathrm{bcd}$ & $29.1 \mathrm{cde}$ & -1.71 \\
\hline G10 & $1.56 \mathrm{ab}$ & $18.96 \mathrm{abc}$ & $24.87 \mathrm{cde}$ & $37.08 \mathrm{~cd}$ & $27.6 \mathrm{def}$ & -6.67 \\
\hline G11 & $1.35 \mathrm{ab}$ & $19.69 \mathrm{ab}$ & $22.13 \mathrm{e}$ & $43.23 \mathrm{bc}$ & $27.0 \mathrm{efg}$ & -8.81 \\
\hline G12 & $1.37 \mathrm{ab}$ & $16.63 \mathrm{cde}$ & $24.80 \mathrm{cde}$ & $50.8 \mathrm{a}$ & $34.0 \mathrm{ab}$ & 14.95 \\
\hline G13 & $1.4 \mathrm{ab}$ & $20.86 \mathrm{a}$ & $32.76 \mathrm{a}$ & $40.78 \mathrm{bcd}$ & $25.3 \mathrm{gh}$ & -14.42 \\
\hline Abdane & $1.44 \mathrm{ab}$ & $14.39 \mathrm{e}$ & $28.96 \mathrm{a}$ & $42.65 \mathrm{bc}$ & $29.6 \mathrm{cde}$ & 0 \\
\hline G14 & $1.38 \mathrm{ab}$ & $19.79 \mathrm{ab}$ & $25.85 \mathrm{bcd}$ & $42.68 \mathrm{bc}$ & $30.7 \mathrm{bcd}$ & 3.78 \\
\hline G2 & $1.41 \mathrm{ab}$ & $16.03 \mathrm{cde}$ & $22.49 \mathrm{de}$ & $43.32 \mathrm{bc}$ & $37.5 \mathrm{a}$ & 26.58 \\
\hline G4 & $1.44 \mathrm{ab}$ & $20.28 \mathrm{ab}$ & $24.79 \mathrm{cde}$ & $43.18 \mathrm{bc}$ & $29.7 \mathrm{cde}$ & 0.36 \\
\hline G5 & $1.28 \mathrm{~b}$ & $17.38 \mathrm{bcd}$ & $24.71 \mathrm{cde}$ & $44.24 \mathrm{ab}$ & $31.6 \mathrm{bc}$ & 6.84 \\
\hline G6 & $1.71 \mathrm{a}$ & $17.99 \mathrm{a}-\mathrm{d}$ & $24.58 \mathrm{cde}$ & $40.94 \mathrm{bcd}$ & $29.6 \mathrm{cde}$ & 0.03 \\
\hline G7 & $1.36 \mathrm{ab}$ & $15.88 \mathrm{de}$ & $26.66 \mathrm{bc}$ & $40.6 \mathrm{bcd}$ & $28.4 \mathrm{c}-\mathrm{f}$ & -3.94 \\
\hline G9 & $1.53 \mathrm{ab}$ & $16.19 \mathrm{cde}$ & $23.37 \mathrm{cde}$ & $35.03 \mathrm{~d}$ & $23.5 \mathrm{~g}$ & -20.77 \\
\hline Local & $1.27 \mathrm{~b}$ & $17.82 \mathrm{bcd}$ & $23.82 \mathrm{cde}$ & $40.76 \mathrm{bcd}$ & $28.2 \mathrm{c}-\mathrm{f}$ & -4.82 \\
\hline M \pm SEM & $1.41 \pm 0.02$ & $17.85 \pm 0.06$ & $25.32 \pm 0.12$ & $42.13 \pm 0.1$ & $29.29 \pm 0.72$ & \\
\hline CV\% & 40.87 & 25.17 & 22.08 & 25.31 & 19.33 & \\
\hline R $\%$ & 0.39 & 0.49 & 0.63 & 0.64 & 79.3 & \\
\hline LSD 5\% & 0.3804 & 2.95 & 3.68 & 7.01 & 3.724 & \\
\hline F test & ns & $* *$ & $* *$ & $* *$ & $* *$ & \\
\hline
\end{tabular}

Key: G-genotype, M-mean DH-Days to Heading; DM-Days to Maturity; PTL-productive tillers, LDG-lodging, PH- Plant

Height; SL-spike Length; SWPP-Spike Weight per plant, SPS-spike lets per spike, GPS-Grain per spike, TSWThousand Seed Weight, QTHA- Yield quintal per Hectare, YAD- yield advantage, CV-Coefficient of variation, $R^{2}$-R-squere, $L S D$ - least significant.

\subsection{Major Disease Reaction of Food Barley Genotypes Across Environments}

Most genotypes evaluated had significantly low scores with their corresponding economically important disease reactions. However, genotypes (G1, G5, Dinsho and Abdane) were less tolerance to scald disease. Similarly, genotypes (G11, G5 G7 and Abdane), were less tolerance to septoria and stem rust. On the other hand, genotypes (G12 and G2) were better tolerance to scald, septoria, stem and leaf rust (Table 5).

Table5. Combined mean of disease reactions (1-5 scale) of food barley genotypes evaluated in 2017-2018 main cropping season.

\begin{tabular}{|c|c|c|c|c|}
\hline Geno/Vrt & SCALD & SEP & SR & LR \\
\hline Dinsho & $1.6 \mathrm{abc}$ & $1.3 \mathrm{bcd}$ & $1.2 \mathrm{f}$ & $1.3 \mathrm{fg}$ \\
\hline G1 & $1.6 \mathrm{a}$ & $1.3 \mathrm{bcd}$ & $1.5 \mathrm{abc}$ & $1.4 \mathrm{ef}$ \\
\hline G10 & $1.3 \mathrm{bcd}$ & $1.4 \mathrm{bc}$ & $1.6 \mathrm{a}$ & $1.6 \mathrm{a}-\mathrm{d}$ \\
\hline G11 & $1.2 \mathrm{~d}$ & $1.4 \mathrm{ab}$ & $1.4 \mathrm{de}$ & $1.5 \mathrm{cde}$ \\
\hline G12 & $1.3 \mathrm{~d}$ & $1.2 \mathrm{de}$ & $1.2 \mathrm{f}$ & $1.3 \mathrm{fg}$ \\
\hline G13 & $1.4 \mathrm{a}-\mathrm{d}$ & $1.3 \mathrm{cde}$ & $1.4 \mathrm{cde}$ & $1.5 \mathrm{de}$ \\
\hline Abdane & $1.6 \mathrm{ab}$ & $1.4 \mathrm{ab}$ & $1.6 \mathrm{a}$ & $1.7 \mathrm{ab}$ \\
\hline G14 & $1.3 \mathrm{bcd}$ & $1.2 \mathrm{e}$ & $1.4 \mathrm{bcd}$ & $1.3 \mathrm{~g}$ \\
\hline G2 & $1.4 \mathrm{a}-\mathrm{d}$ & $1.4 \mathrm{abc}$ & $1.4 \mathrm{cde}$ & $1.4 \mathrm{efg}$ \\
\hline G4 & $1.3 \mathrm{a}-\mathrm{d}$ & $1.3 \mathrm{~b}-\mathrm{e}$ & $1.4 \mathrm{de}$ & $1.5 \mathrm{cde}$ \\
\hline G5 & $1.6 \mathrm{abc}$ & $1.5 \mathrm{a}$ & $1.5 \mathrm{ab}$ & $1.8 \mathrm{a}$ \\
\hline G6 & $1.4 \mathrm{a}-\mathrm{d}$ & $1.3 \mathrm{~b}-\mathrm{e}$ & $1.3 \mathrm{ef}$ & $1.6 \mathrm{~b}-\mathrm{d}$ \\
\hline G7 & $1.3 \mathrm{~cd}$ & $1.4 \mathrm{ab}$ & $1.4 \mathrm{cde}$ & $1.4 \mathrm{efg}$ \\
\hline G9 & $1.4 \mathrm{a}-\mathrm{d}$ & $1.3 \mathrm{~b}-\mathrm{e}$ & $1.6 \mathrm{a}$ & $1.6 \mathrm{abc}$ \\
\hline Local & $1.3 \mathrm{a}-\mathrm{d}$ & $1.3 \mathrm{cde}$ & $1.6 \mathrm{a}$ & $1.5 \mathrm{de}$ \\
\hline Mean \pm SEM & $1.40 \pm 0.04$ & $1.33 \pm 0.02$ & $1.42 \pm 0.03$ & $1.49 \pm 0.03$ \\
\hline
\end{tabular}

International Journal of Research Studies in Agricultural Sciences (IJRSAS)

Page $\mid 10$ 
Multi-Location Evaluation of Yield And Yield Related Trait Performance In Food Barley (Hordeum Vulgare L.) Genotypes at Western Oromia, Ethiopia

\begin{tabular}{|c|c|c|c|c|}
\hline CV\% & 32.99 & 16.08 & 14.08 & 16.58 \\
\hline $\mathrm{R}^{2} \%$ & 64 & 77 & 89 & 86 \\
\hline LSD 5\% & 0.3 & 1.97 & 0.13 & 0.16 \\
\hline F test & $* *$ & $* *$ & $* *$ & $* *$ \\
\hline
\end{tabular}

Key: G-genotype, CV-Coefficient of variation, LSD- least significant difference, $\mathrm{R}^{2}$-R-Squere, SR-stem rust, LR-leaf rust, SEP-septoria, SEM- standard error of mean .1-5 scale where 1= resistant, 5= susceptible

\subsection{AMMI Analysis for Grain Yield}

The additive main effects and multiplicative interaction analysis (Table 6) of grain yield showed that environment, and genotypes by environment interaction were highly significant $(\mathrm{P} \leq 0.01)$. This is similar to the report of [23]. This indicates that one of the basic factors that affect GEI could either be genotypic or environmental in nature $[8,2]$ also reported that $74.3 \%$ of the interaction sum of squares was explained by IPCA1.

Table6. Additive main effect and multiplicative interaction analysis of variances (AMMI) for grain yield of 15 food barley genotypes evaluated at six environments

\begin{tabular}{|c|c|c|c|c|}
\hline Source of variation & DF & SS & EX.SS\% & MS \\
\hline Total & 269 & 27580 & 100 & $245.3^{* *}$ \\
\hline Treatments & 89 & 21830 & 79.15 & $206.1^{* *}$ \\
\hline Genotypes & 14 & 2886 & 10.46 & $2261.7^{* *}$ \\
\hline Environments & 5 & 11308 & 41.00 & $30.1^{\mathrm{ns}}$ \\
\hline Block & 12 & 361 & 1.31 & $241.1^{* *}$ \\
\hline GEI & 70 & 7636 & 27.69 & $98.4^{* *}$ \\
\hline IPCA 1 & 18 & 4340 & 15.74 & 47.8 \\
\hline IPCA 2 & 16 & 1575 & 5.71 & 32.1 \\
\hline Residuals & 36 & 1721 & 6.24 & \\
\hline Error & 168 & 5390 & & Principal \\
\hline
\end{tabular}

Key: $D F=$ degree of freedom, $S S=$ sum of squares, $M S=$ mean squares, $I P C A=$ Interaction Principal Component Axis, EX.

SS\% = Explained Sum of square $n *^{*}, * *$ non-significant, Significant at the $5 \%$ and $1 \%$ level of probability respectively

The environment and genotype mean are presented in Fig.1 and Table 7. This bi-plot helped in the interpretation of the interaction effects among genotypes and environments; and in the assessment of the adaptability of genotypes. Genotype (G12 and G2) with a lower IPCA1 score were stable genotypes. Genotypes which are characterized by mean greater than grand mean and the IPCA scores nearly zero are considered as generally adaptable to all environment. However, the genotypes with high mean performance and with large value of IPCA scores are considered as having specific adaptability to the environments [26]. Study by [5] reported that the larger the IPCA scores, either negative or positive, the more specifically adapted a genotype is to a certain environments; yet the smaller the IPCA scores, the more stable the genotype is over all environments. Genotypes (G14 and G4) had grain yield above the grand mean; and similar IPCA1 scores with locations Bedesso and Belem implying that their interactions were positive; the higher yields of these genotypes were found, particularly, at these locations. Hence, they were the best adapted genotypes for these locations.

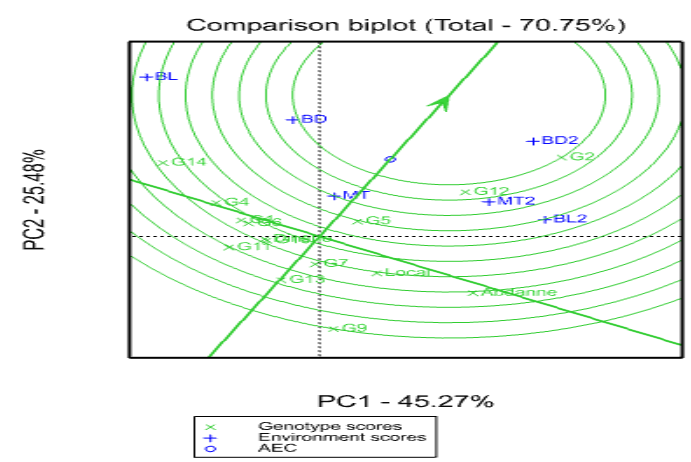

Figure1. GGE bi-plot based on genotype-focused scaling for comparison of genotypes for their yield potential and stability 
Multi-Location Evaluation of Yield And Yield Related Trait Performance In Food Barley (Hordeum Vulgare L.) Genotypes at Western Oromia, Ethiopia

Key: G-genotypes, BD-bedesso, BL-Belem, MT-Mata, Number followed the lactations indicate the year $(1=2017,2=2018)$

Table7. Mean grain yield $\left(Q\right.$ tha $\left.^{-1}\right)$ per location and year from the AMMI additive GE model

\begin{tabular}{|c|c|c|c|c|c|c|c|c|c|}
\hline Genotype & $\begin{array}{c}\text { BD1 } \\
(\mathrm{E} 1)\end{array}$ & $\begin{array}{c}\text { BD2 } \\
(\mathrm{E} 2)\end{array}$ & $\begin{array}{c}\text { BL1 } \\
(\mathrm{E} 3)\end{array}$ & $\begin{array}{c}\text { BL2 } \\
(\mathrm{E} 4)\end{array}$ & $\begin{array}{c}\text { MT1 } \\
(\mathrm{E} 5)\end{array}$ & $\begin{array}{c}\text { MT2 } \\
(\text { E6 })\end{array}$ & Mean & IPCAg1 & IPCAg2 \\
\hline Abdane & 10.46 & 37.1 & 10.94 & 46.12 & 33.92 & 39.3 & $\mathbf{2 9 . 6 4}$ & 2.72 & -1.89 \\
\hline Dinsho & 20.32 & 31.73 & 23.58 & 32.18 & 24.62 & 32.38 & $\mathbf{2 7 . 4 7}$ & -0.66 & 0.69 \\
\hline G1 & 19.71 & 29.86 & 28 & 33.17 & 31.85 & 31.97 & $\mathbf{2 9 . 0 9}$ & -1.32 & -0.7 \\
\hline G10 & 21.38 & 32.55 & 23.63 & 32.07 & 23.29 & 32.83 & $\mathbf{2 7 . 6 3}$ & -0.63 & 1.09 \\
\hline G11 & 19.14 & 29.32 & 25.11 & 30.93 & 26.77 & 30.67 & $\mathbf{2 6 . 9 9}$ & -1.13 & 0.07 \\
\hline G12 & 23.97 & 43.49 & 20.55 & 44.63 & 28.26 & 43.24 & $\mathbf{3 4 . 0 2}$ & 1.55 & 1.06 \\
\hline G13 & 17.71 & 31.61 & 18.29 & 31.81 & 20.78 & 31.79 & $\mathbf{2 5 . 3 3}$ & 0.07 & 1.01 \\
\hline G14 & 29.28 & 30.95 & 37.27 & 28.11 & 27.12 & 31.59 & $\mathbf{3 0 . 7 2}$ & -3.05 & 1.36 \\
\hline G2 & 28.39 & 50.62 & 19.86 & 49.83 & 27 & 49.11 & $\mathbf{3 7 . 4 7}$ & 2.52 & 2.14 \\
\hline G4 & 22.37 & 29.97 & 31.08 & 31.81 & 31.21 & 31.81 & $\mathbf{2 9 . 7 1}$ & -1.88 & -0.25 \\
\hline G5 & 16.82 & 33.99 & 23.64 & 41.12 & 37.43 & 36.75 & $\mathbf{3 1 . 6 2}$ & 0.25 & -1.83 \\
\hline G6 & 17.56 & 28.93 & 28.15 & 34.82 & 36.19 & 32 & $\mathbf{2 9 . 6 1}$ & -1.25 & -1.75 \\
\hline G7 & 14.86 & 31.35 & 20.78 & 37.32 & 32.58 & 33.7 & $\mathbf{2 8 . 4 3}$ & 0.18 & -1.36 \\
\hline G9 & 12.36 & 31.76 & 10.64 & 34.09 & 19.8 & 32.06 & $\mathbf{2 3 . 4 5}$ & 1.39 & 0.51 \\
\hline Local & 15.69 & 35.14 & 15.98 & 38.99 & 27.14 & 36.11 & $\mathbf{2 8 . 1 7}$ & 1.24 & -0.16 \\
\hline Environment & & & & & & & & & \\
\hline Mean & $\mathbf{1 9 . 3 3}$ & $\mathbf{3 3 . 8 9}$ & $\mathbf{2 2 . 5}$ & $\mathbf{3 6 . 4 7}$ & $\mathbf{2 8 . 5 3}$ & $\mathbf{3 5 . 0 2}$ & $\mathbf{2 9 . 2 9}$ & & \\
\hline IPCAe1 & -1.71 & 2.10 & -4.34 & 2.81 & -0.71 & 1.85 & & & \\
\hline IPCAe2 & 2.42 & 1.51 & 0.06 & -0.89 & -3.69 & 0.58 & & & \\
\hline
\end{tabular}

G-genotypes, E-environment, BD-bedesso, BL-Belem, MT-Mata, IPAg-interaction principal axis to genotypes, IPAe-interaction principal axis to environment, Number followed the lactations indicate the year $(1=2017,2=$ 2018)

\subsection{Stability Analysis for Genotypic Performance}

AMMI Stability Value (ASV). In table 8 shows, AMMI stability values for grain yield. considering the AMMI stability value (ASV) that takes into account the scores of the IPCA2, genotypes with least ASV scores are the most stable, whereas genotypes with high ASV score are unstable [11, 5, 17]. Accordingly, genotypes (Dinsho, G11 and G13) were appeared to be among those showing low ASV and were the most stable. On the contrary, genotypes G14 and Abdane revealed the highest ASV and were thus considered to be unstable. Stability by itself should, however, not be the only parameter for selection, as the most stable genotype would not necessarily give the best yield performance [28]. Therefore, the study indicated that, Dinsho, G11 and G13 were recorded the lower ASV (Table 8), but recorded lower yield (27.47, 26.99 and $25.33 \mathrm{Qtha}^{-1}$ respectively) than the grand means (29.29 $\left.\mathrm{Qtha}^{-1}\right)$. So if Dinsho, G11 and G13 will be selected based on ASV per se, there will be a risk of yield reduction. The stable genotype was followed with mean grain yield above the grand mean and this result was in agreement with [15], who has used ASV as one method of evaluating grain yield stability of bread wheat varieties in Tigray and similar reports been made by [4], [27] in barley in Tigray and bread wheat using AMMI stability value. A genotype with the least of genotype selection index (GSI) is considered as the most stable genotype [11]. Accordingly, G2, G12 and G5 were more stable genotypes with the low of genotype selection index (GSI) and higher mean grain yield (Table 8).

Table8. AMMI stability value, AMMI rank, Yield, yield rank and genotype selection index (GSI)

\begin{tabular}{|c|c|c|c|c|c|}
\hline Genotype & ASV & ASV RANK & YLD & YLD RANK & GSI \\
\hline G2 & 5.49 & 13 & $\mathbf{3 7 . 4 7}$ & 1 & 14 \\
\hline G12 & 3.12 & 10 & $\mathbf{3 4 . 0 2}$ & 2 & 12 \\
\hline G5 & 3.07 & 9 & $\mathbf{3 1 . 6 2}$ & 3 & 12 \\
\hline G14 & 5.54 & 15 & $\mathbf{3 0 . 7 2}$ & 4 & 19 \\
\hline G4 & 3.15 & 11 & $\mathbf{2 9 . 7 1}$ & 5 & 16 \\
\hline Abdane & 5.50 & 14 & $\mathbf{2 9 . 6 4}$ & 6 & 19 \\
\hline G6 & 3.57 & 12 & $\mathbf{2 9 . 6 1}$ & 7 & 16 \\
\hline G1 & 2.48 & 8 & $\mathbf{2 9 . 0 9}$ & 8 & 19 \\
\hline
\end{tabular}

International Journal of Research Studies in Agricultural Sciences (IJRSAS)

Page | 12 
Multi-Location Evaluation of Yield And Yield Related Trait Performance In Food Barley (Hordeum Vulgare L.) Genotypes at Western Oromia, Ethiopia

\begin{tabular}{|c|c|c|c|c|c|}
\hline G7 & 2.28 & 6 & $\mathbf{2 8 . 4 3}$ & 9 & 15 \\
\hline Local & 2.08 & 4 & $\mathbf{2 8 . 1 7}$ & 10 & 14 \\
\hline G10 & 2.09 & 5 & $\mathbf{2 7 . 6 3}$ & 11 & 16 \\
\hline Dinsho & 1.59 & 1 & $\mathbf{2 7 . 4 7}$ & 12 & 13 \\
\hline G11 & 1.88 & 3 & $\mathbf{2 6 . 9 9}$ & 13 & 16 \\
\hline G13 & 1.68 & 2 & $\mathbf{2 5 . 3 3}$ & 14 & 16 \\
\hline G9 & 2.46 & 7 & $\mathbf{2 3 . 4 5}$ & 15 & 22 \\
\hline
\end{tabular}

G- genotype

\section{CONCLUSION AND RECOMMENDATIONS}

Although the GEI of grain yield partitioned in to different IPCAs using AMMI model analysis, the first principal component axis for interaction alone explains most of the interaction sum of squares. The sign and magnitude of IPCA scores showed the relative contribution of each genotype and environment for the genotype and environment interactions. It helps to summarize the pattern and magnitude of GEI and main effects that reveal clear insight into the adaptation of genotypes to environments. This shows that genotypes (G2 and G12) are less contributors to the interaction effect and have consistent performances across locations whereas genotype, G14 with higher ASV scores and unstable genotype. Therefore, G2, and G12 were identified as the best genotypes in terms of yielding ability and stability, tolerant to diseases for advancement, release and use as parents in future breeding programs.

\section{ACKNOWLEDGEMENT}

The authors greatly acknowledged Oromia Agricultural Research Institute (IQQO) for financial support. Haro-Sebu Agricultural Research Center staff members are warmly acknowledged for technical and administrative support. Particularly, all cereal research team members significantly thankful for their technical support and field work. And as well Ethiopia biodiversity institute (EBI) and Sinana agricultural research center are acknowledged for the provision of test materials.

\section{REFERENCES}

[1] Bayeh M., Berhane L., Barley research and development in Ethiopia - an overview. In:BayehM.,GrandoS(eds.),Barley Research and Development in Ethiopia. Proceedings of the $2^{\text {nd }}$ National Barley Research and Development Review Workshop, Holetta Agricultural Research Centre, Ethiopia 28 30 November 2006, HARC, Holetta, Ethiopia. ICARDA, Aleppo, Syria. Pp.1-18 (2011).

[2] Anandan A., Eswaran R., Sabesan T. and Prakash M., Additive main effects and multiplicative interactions analysis of yield performances in rice genotypes under coastal saline environments, Indian Journal of Advances Biological Research 3(1-2), 43-47 (2009).

[3] Asrat A., Fekadu G. and Mulugeta A., "AMMI and SREG GGE biplot analysis for matching varieties onto soybean production environments in Ethiopia." Scientific Research and Essay, vol. 4, pp. 1322-1330 (2009).

[4] Abay F. and Bjørnstad A., Specific adaptation of barley varieties in different locations in Ethiopia. Euphotic, 167: 181-195 (2009)

[5] Bantayehu M.,Analysis and correlation of stability parameters in malting barley. African Crop Science Journal 17 (3),145-153 (2009)

[6] Comstock RE. and Moll RH., Genotype x Environment Interactions. Symposium on Statistical Genetics and Plant Breeding. National Academy Science National Research Council, Washington, D.C. pp. 164-196 (1963).

[7] CSA (Central Statistics Authority), The Federal Democratic Republic of Ethiopia, Central Statistical Agency agricultural sample survey 2016/2017 (2009 E.C) volume I report on area and production of major crops (private peasant holdings, Meher season). Statistical Bulletin no.584, Addis Ababa, Ethiopia, pp. 118. (2017).

[8] Debelo D., Girma B., Alemayehu Z. and Gelalcha S., Drought tolerance of some bread wheat genotypes in Ethiopia. African Crop Science Journal 9 (2), 385-392 (2000).

[9] Delacy IH., Basford KE., Cooper M. and Bull JK., Analysis of multi- environment trials- an historical perspective. Plant Adaptation and Crop Improvement. Eds. M. Cooper and G. L. Hammer. CAB international, pp. 39-124 (1996).

[10] FAO (Food and Agriculture Organization) Food Balance Sheets FAOSTAT Database on Agriculture (2014).

[11] Farshadfar E., Incorporation of AMMI stability value and grain yield in a single nonparametric index (GSI) in bread wheat. Pakistan Journal of Biological Sciences 11:1791-1796 (2008). 
[12] Gabriel KR., The bi-plot graphic of matrices with application to principal component analysis. Biometrics 58:453-467 (1971)

[13] Gauch HG., Statistical analysis of yield trials by AMMI and GGE. Crop Sciences. 46:1488-1500 (2006).

[14] Harlan JR., Evolution of Crop Plants. New York. N.W Simmonds (eds.), University of Illinois Urbana Iii USA, 2008. Longman.

[15] Hintsa G. and Abay F., Evaluation of Bread Wheat Genotypes for their Adaptability in wheat growing Areas of Tigray Region, northern Ethiopia. Journal of Biodiversity and Endangered Species, (2013).

[16] Horsley RD. and Hochhalter M., Barley Agronomy. Encyclopedia of Grain Science, Wrigley C, Corke H, Walker H. (eds.). Elsevier Academic press, (2004).

[17] Issa A.B., Genotype by environment interaction and yield stability of maize hybrids evaluated in Ethiopia. MSc. in Agriculture, Free State University, South Africa, Pp.121, (2009).

[18] Tamene T., Gemechu K., Tadese S., Mussa J. and Yeneneh B.,"Genotype x environment interaction and performance stability for Grain Yield in Field Pea (Pisumsativum L.) Genotypes." International Journal of Plant Breeding, vol. 7, pp. 116-123 (2013)

[19] VSN International, "Gen Stat for Windows 15th Edition. VSN International, Hemel Hempstead, UK." Available: www.genStat.co.uk (2012).

[20] Yan W., Hunt LA., Sheng Q. and Szlavnics Z., Cultivar evaluation and mega environment investigation based on the GGE bi-plot. Crop Science.40:597-605 (2000)

[21] Yan W. and Kang MS., GGE bi-plot analysis: a graphical tool for breeders, In Kang MS, ed. Geneticists, and Agronomist. CRC Press, Boca Raton, FL, pp. 63-88 (2003).

[22] Yan W., GGE bi-plot- a windows application for graphical analysis of multi-environment trial data and other types of two-way data. Journal of Agronomy 93:1111-1118 (2001).

[23] Ntawuruhunga P.H., Rubaihayo P., Whyte J.B.A., Dixon A.G.O. and Osiru D.S.O., Additive main effects and multiplication interaction analysis for storage root yield of cassava genotypes evaluated in Uganda. Africa Crop Sciences Journal 9 (4), 591-598 (2001).

[24] Purchase JL., Parametric analysis to describe genotype $x$ environment interaction and yield stability in winter wheat. Ph.D. Thesis, Department of Agronomy, Faculty of Agriculture of the University of the Free State, Bloemfontein, South Africa (1997).

[25] SAS Institute Inc., Statistical analysis Software version 9.2, Cary, NC: SAS Institute Inc. USA (2008).

[26] Singh D., Singh S.K. and Singh K.N., AMMI analysis for salt tolerance in bread wheat genotypes.www.cabdirect.org/abstracts/ 20093259042.html.Accessed on 10 February 2011. (2009).

[27] Sivapalan S., Obrien L., Ortiz-Ferrara G., Hollamby GJ., Barclay I. and Martin PJ., An adaptation analysis of Australian and CIMMYT/ICARDA wheat germplasm in Australian production environments. Crop Science pastures 51: 903-915 (2000).

[28] Mohammadi R., Armion M., Shabani A. and Daryaei A.,Identification of stability and adaptability in advanced durum wheat genotypes using AMMI analysis. Asian Journal of Plant Sciences 6:1261-1268 (2007).

[29] Kang MS., Breeding: Genotype-by-environment interaction. In R.M. Goodman (ed.) Encyclopedia of plant and crop science. Marcel- Dekker, New York. pp. 218-221. (2004).

[30] Kaya Y., Akcura M. and Taner S., GGE-bi-plot analysis of multi- environment yield trials in bread wheat. Turkish Journal of Agriculture 30:325- 337 (2006).

[31] Martin JH. and Leonard WH., Principles of Field Crops Production. $2^{\text {nd }}$ ed. London, MacMillan Company, (2010).

[32] Mitrovic B., Stanisavljevi D., Treski S., Stojakovic M., Ivanovic M., Bekavac G. and Rajkovic M., Evaluation of experimental Maize hybrids tested in Multi-location trials using AMMI and GGE bi-plot analysis. Turkish Journal of Field Crops, 17:35-40. (2012).

Citation: Geleta Negash, et.al. "Multi-Location Evaluation of Yield and Yield Related Trait Performance in Food Barley (Hordeum Vulgare L.) Genotypes at Western Oromia, Ethiopia” International Journal of Research Studies in Agricultural Sciences (IJRSAS), 2019; 5(5), pp. 5-14, http://dx.doi.org/10.20431/24546224.0505002

Copyright: (C) 2019 Authors. This is an open-access article distributed under the terms of the Creative Commons Attribution License, which permits unrestricted use, distribution, and reproduction in any medium, provided the original author and source are credited. 